\title{
Three Dimensional Computer Vision for Computer Aided Design and Manufacturing Applications
}

\author{
M. Milanova, L. Nikolov, Svetlin Fotev* \\ Department of Radiocommunication \\ Technical University \\ 83 Simeon Str. \\ Sofia 1000 \\ Bulgaria \\ e-mail: mariofanna.milanova@infolink.infotel.bg \\ * Bulgarian Academy of Sciences \\ Space Research Institute \\ 6, Moskovska str. \\ 1000 , Sofia \\ Bulgaria
}

\begin{abstract}
Our work focuses on the problem of automatic generation of viewpoint invariant geometric surface models from range data to be used in CAD/CAM systems. We developed two methods for recovering the surface. First is on the base of Shape from Shading method and second is on the base of Facet method. A comparison between the results obtained using the 2 methods is made. We also offer new techniques for the representation and $\mathrm{CNC}$ processing of the surfaces.
\end{abstract}

\section{Introduction}

Computer vision systems are gaining wide acceptance in industrial applications such as design automation and manufacturing automation. The problem of linking CAD and Vision systems is very difficult (Besl P,1985). We have to decide two important tasks:

1. How to gather reliable information about the 3-D object of the scene.

2. How to represent 3-D object for both CAD and Computer Vision applications.

We propose surface based segmentation and parametric surface description of complex 3D objects liable to automatic mechanical process. The description of visible surfaces obtained by their recovering is used directly in the CAD/CAM systems where the of fset of the object is determined easily and the tool path is simulated.

\section{Methods for recovering of the objects}

Two methods are investigated for oblaining surface characteristics from intensity images in this work.

\subsection{Modified Shape from Shading method}

First we use and modify Shape from Shading method and obtain range data information.

We developed an algorithm for recovering three-dimensional shape of visible surfaces in a scene from a single two-dimensional image on the base of iteralive method (Shirai Y.,1988). We restricted our attention to scenes composed of one opaque solid objecl bounded by piecewise smooth surfaces with no markings. 
The relationship between the shape of the object determined by the normal vector $(f, g)$ and the brightness $E$ in the respective point of the image can be expressed by the following equation:

$f=2 p /\left(\sqrt{1+p^{2}+q^{2}}\right) ; \quad g=2 q /\left(\sqrt{1+p^{2}+q^{2}}\right)$

where $f=2 p /\left(\sqrt{1+p^{2}+q^{2}}\right) ; \quad g=2 q /\left(\sqrt{1+p^{2}+q^{2}}\right)$

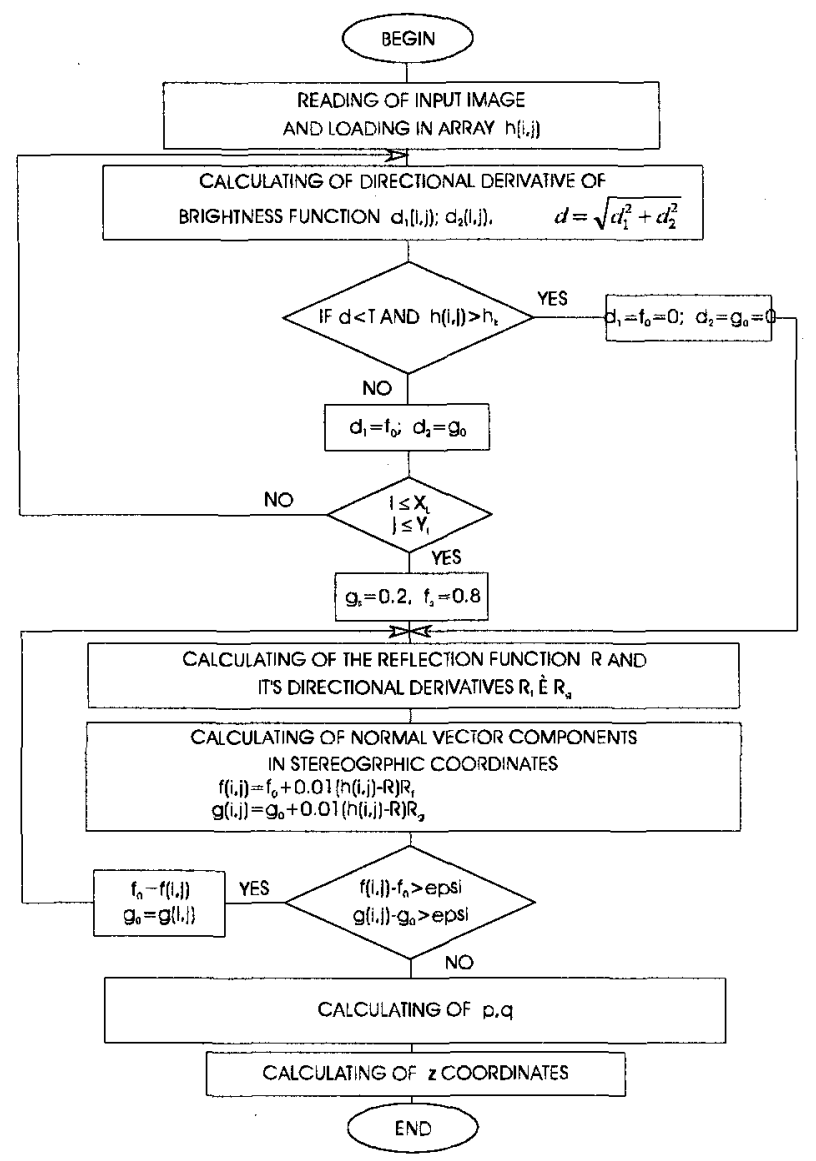

Fig.1. The flowchart of modified Shape from Shading method

We seek a set of values $\left\{f_{1, j}\right\}$ and $\left\{g_{1}\right\}$ that minimize the total error.

$e=\sum_{i=1}^{n} \sum_{j=1}^{m}\left(s_{i, j}+\lambda r_{i, j}\right)$

where:

$s_{i j}$ is the error which measures the departure from smoothness at the point $(i, j)$. $r_{1 j}$ is the error in the image irradiance equation. 
Equation (2) is minimized by differentiating with respect to $f$ and $g$. To find $f$ and $g$ we use the relaxation process:

$f_{k 1}{ }^{n+1}=f_{k 1}{ }^{n}-\lambda\left[E_{k 1}-R\left(f_{k 1}, g_{k 1}\right)\right] d R / d f$

$\mathrm{g}_{\mathrm{k} !}{ }^{\mathrm{n}+1}=\mathrm{g}_{\mathrm{k} 1}{ }^{\mathrm{n}}-\lambda\left[\mathrm{E}_{\mathrm{k} 1}-\mathrm{R}\left(\mathrm{f}_{\mathrm{k} 1}, \mathrm{~g}_{\mathrm{k} 1}\right)\right] \mathrm{dR} / \mathrm{dg}$

Then we create the needle diagram.

We now wish to recover the surface $\left\{z_{1 j}\right\}$. To do this, we use an iterative method based on the following relaxation process:

$z_{k, 1}^{n+1}=h / 4\left[\left(p_{k, 1-1}-p_{k, 1}+p_{k-1,1-1}-p_{k-1,1}\right)+\left(q_{k-1,1}-q_{k, 1}+q_{k-1,1-1}-q_{k, 1-1}\right)\right]$ $+1 / 4\left(z_{k-1,1-1}^{n}+z_{k-1,1+1}^{n}+z_{k+1,1+1}^{n} z_{k-1,1-1}^{n}\right)$

The flowchart of presented algorithm is shown in Figure 1. After segmentation, we can obtain the area of the object and then apply edge detection to find its boundary which is composed of the zero-crossing pixels. The plane constituted by these pixels is the reference plane of the depth map.

The resulting reconstruction often is only qualitatively reasonable and may not have desired numerical accuracy primarily due to the following reasons (Berthold K., 1993; Bhandarkar S.M., 1992):

1) the constraints available to us here are the values of $f$ and $g$ on the occluding boundary;

2) The Lambertian model may not be an accurate model for the image surfaces;

3) interreflections and specularities may also cause inaccurate reconstruction.

The originality of this method is in the using of additional information about the boundary of the surface. An algorithm for contour segmentation and an algorithm for determining the boundary are applied. The direction of the normal vectors at every point of the boundary is calculated in stereographic coordinates.

\subsection{Facet method}

The second investigated method is on the base of Haralick facel method (Haralick R. 1983). In this method a physical surface in an image is typically represented by many image facets. It describes the shape of the gray level intensity surface for each pixel and assemblies all the shape fragments to reconstruct the entire surface of the image gray level intensity values.

The gray tone in each facel must be a polynomial function of the row-column coordinates of the pixels in the facet. In each neighbourhood the gray tone intensity function $f$ has the form:

$f(r, c)=k_{1}+k_{2} r+k_{3} c+k_{4} r^{2}+k_{5} r c+k_{6} c^{2}+k_{7} r^{3}+k_{8} r^{2} c+k_{9} r c+k_{10} c^{3}$

The flowchart of presented algorithm is shown in Figure 2.

\section{Manufacturing of sculptured surfaces using CNC machines}

One of the most complex aspects of CAM is the manufacture of sculptured surfaces. The basic problems which have to be solved in the process of surface manufacture are:

1. Generating the mathematical representation of the surface;

2. Generaling the tool path, i.e. converting the surface representation into a sequence of linear culter paths; 


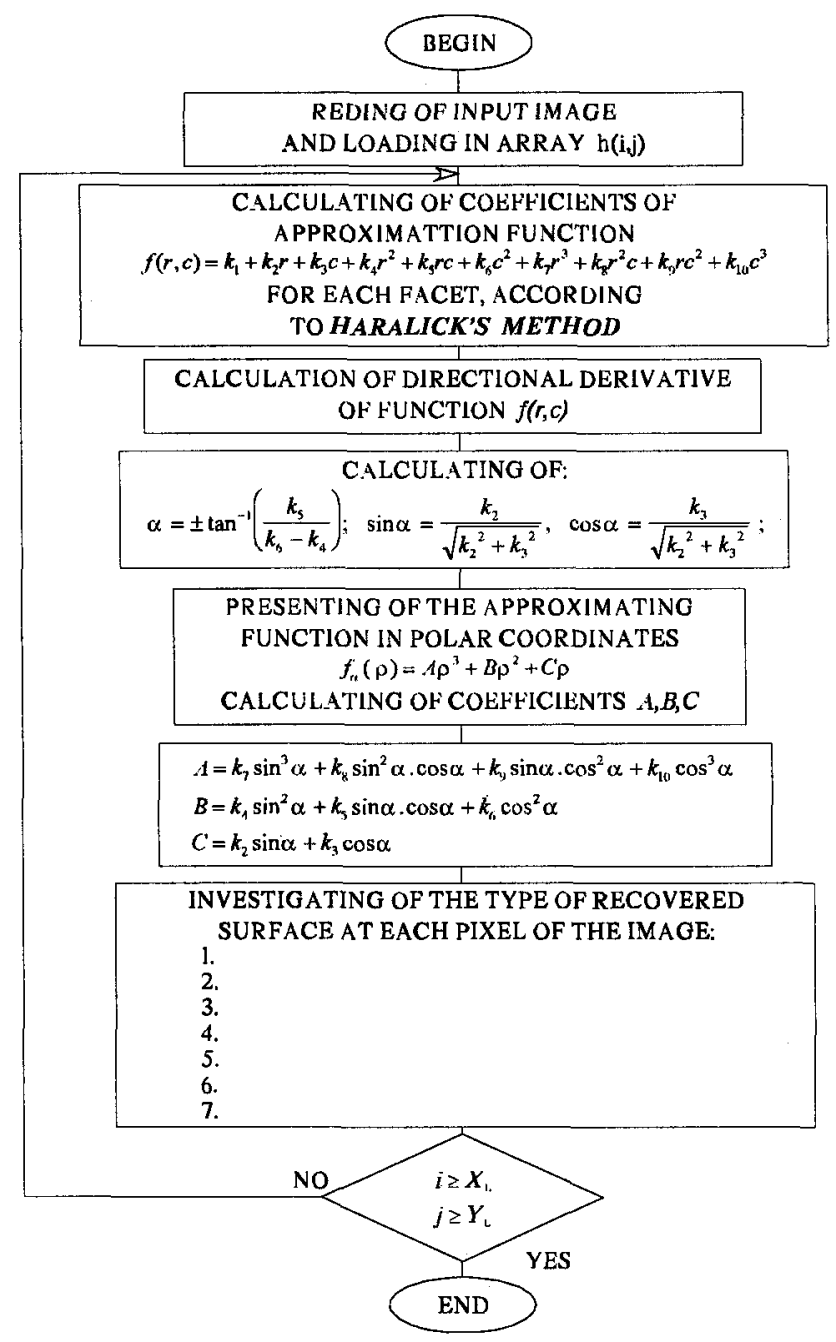

Fig.2. The flowchart of modified Facet method

3. Controlling the tool to move along prescribed cutter paths on multi-axis machines. In this paper a new path generation method is proposed. The surface which is desired to be manufactured is represented as an integration of parts of other surfaces. Every of these surfaces is represented as a rectangular mesh of points.

\subsection{Mathematical description of the surface}

Given the output of the algorithm discussed in the preceding section a graph surface form is created $(z=f(x, y))$. In this section, we will discuss a parametric surface reconstruction scheme that is invariant to three dimensional rigid body motion. The received mesh of points is approximated by a mesh of curves. The mesh of cubic interpolation splines is chosen because it can be calculated easily and it passes through all the data points. 
Let $\left(\mathrm{x}_{0}, \mathrm{y}_{0}, \mathrm{z}_{0}\right), \ldots . .,\left(\mathrm{x}_{\mathrm{n}}, \mathrm{y}_{\mathrm{n}}, \mathrm{z}_{\mathrm{n}}\right)$ be the points belonged to one of the lines of the surface (u- or $\mathrm{v}$ line). Let's consider the coordinate $x$ of an arbitrary point of the line (the other coordinates are obtained in the same way). Let $x(t)$ be the function which determines the value of $x$ coordinate according to the parameter $t$. Suppose that $t \in[0,1]$ and $x(i / n)=x i(i=0, \ldots, n)$ Then the formula for determining $x(t)$ is the following:

$$
x(t)=M_{i-1} \frac{n(i / n-t)^{3}}{6}+M_{i} \frac{n(t-i /(n-1))^{3}}{6}+\left(x_{i-1}-\frac{M_{i-1}}{6 n^{2}}\right)(i / n-t)+\left(x_{i}-\frac{M_{i}}{6 n^{2}}\right)(t-i /(n-1)
$$

where $t \in[(i-1) / n, i / n], i=0, \ldots, n$.

To determine the coefficients Mi the following system should be solved:

$\frac{M_{i-1}}{6 n}+\frac{2 M_{i}}{3 n}+\frac{M_{i+1}}{6 n}=\left(x_{i+1}-2 x_{i}+x_{i-1}\right) n, i=1, \ldots, n-1$

$\mathrm{M}_{0}=\mathrm{M}_{\mathrm{n}}=0$.

In this way we can oblain the mesh of natural cubic interpolation splines which represents the surface.

\subsection{Determining the step in v-direction}

Let's suppose that the surface will be manufactured by the tool along u-lines one by one. Then the next step is to determine the step in $\mathrm{v}$-direction according to the tool radius $\mathrm{R}$ and scallop height $\mathrm{h}$ so that the surface can be processed wholly.

If $I$ is the step size in the $v$-direction then the following equation is obtained:

$\mathrm{I}=2\left(\mathrm{~h}(2 \mathrm{R}-\mathrm{h})^{1 / 2}\right.$

In order to receive proportional moving in $v$-direction for all $v$-lines the step in the $v$ direction is defined in the following way:

$\operatorname{step}_{\mathrm{v}}=\mathrm{I} / \max _{\mathrm{j}} \mathrm{S}_{\mathrm{j}}$

where $s_{1}, \ldots s_{n}$ are the tolal lengths of the v-lines of the surface.

If $r(u, v)$ represents the coordinates of an arbitrary point of the surface $(u, v \in[0,1])$, then

$s_{j}=\int_{0} \sqrt{\dot{r}^{2}\left(u_{j}, v\right)} d v=\int_{0}^{1} \sqrt{\dot{x}^{2}+\dot{y}^{2}+\dot{z}^{2}} d v$

To calculate this integral the following approximation formula can be used:

$s_{j}=\int_{0} f(v) d v=f(0)+2 \sum_{i=1}^{n-1}[f(i / n)+f(1)] / 2 n$

To calculate

$f(i / n)=x^{2}(i / n)+y^{2}(i / n)+z^{2}(i / n), i=0, \ldots \ldots, n$

we know that $x, y, z$, are cubic interpolation splines:

$$
\begin{aligned}
\dot{x}(v)= & -M_{i-1} \frac{(i / n-v)^{2} n}{2}+M_{i} \frac{(v-(i-1) / n)^{2} n}{2}+ \\
& +n\left(x_{i}-x_{i-1}\right)+\frac{M_{i}-M_{i-1}}{6 n}
\end{aligned}
$$




\subsection{Path generation method}

The aim of this method is to approximate the parametric space curves of a three-dimensional sculptured surface (in this case the curves are u-lines of the surface) by a sequence of linear segments.

The algorithm determines the points recursively. The first point, $P_{0}$ is the beginning of the u-line Figure 3.

Suppose that the point $P_{1}$ has already been determined. Then the next point $P_{1+1}$ of the sequence of points is determined in the following way:

Let's approximate the curve around $\mathrm{Pi}$ to its oscillating circle. Suppose that the Cartesian coordinate values $(x, y, z)$, the unit normal vector $\mathrm{N}$, the unit tangent vector $\mathrm{T}$ and the radius of curvature $r$ are given. As it is shown in (Kim , 1988; Milanova M., 1995), the next segment point $P_{1+1}$ on the curve is evalualed by:

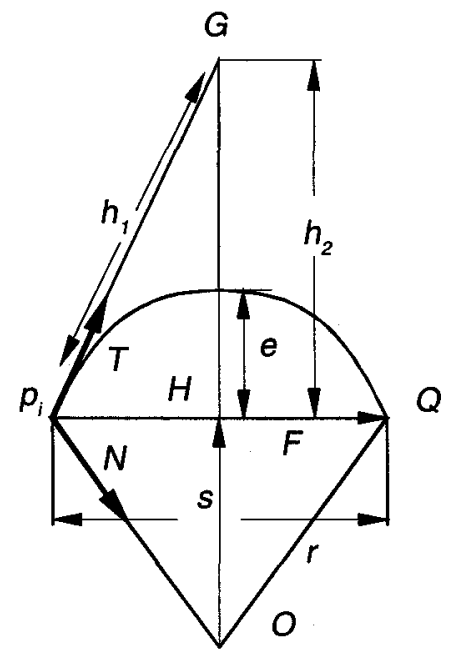

Fig.3. Next point generation

$P_{i+1} \approx Q=P_{i}+\left(\frac{\sqrt{2 e(2 r-e)}(r-e)}{r}\right) \Gamma+\left(\frac{2 e(2 r-e)}{2 r}\right) N$

\subsection{Determining the offset of the surface and creating CNC program}

Since the surface is manufactured by a ball nose cutter the of fset of the tool path should be determined. The cutter off set point $C_{i}$ corresponding to the segment point $P_{\text {, }}$ of a linearly approximated curve of the surface can be given by:

$\mathrm{C}_{\mathrm{i}}=\mathrm{P}_{\mathrm{i}}+\frac{\mathrm{R}}{\text { T.F }} \mathrm{N}$

where $R$ is the radius of the ball-ended cutter and $F$ is the unit vector in the direction $P_{1} P_{1+1}$.

\section{Experiments}

The experimental results are for the images presented on Figure $4 a, 4 b$. These images are 
received by TV camera and a computer system for image processing. The size of the image matrix is $512 \times 512$ and 8 bits per pixel. 256 gray tone levels are used.

Figure 5 shows the result from the method described in 2.2, more precisely, the ridges (white points) and the valley (black points). The sizes of the facets are $7 \times 7$ Figure $5 \mathrm{a}, 11 \times 11$ Figure 5b.

Table 1 presents the error in the recovering of the surfaces using the method explained in 2.1. The error in the recovering of the Z-coordinate is estimated by the following expression:

$\overline{\mathrm{e}}_{\mathrm{z}}^{2}=\frac{1}{\mathrm{~N}^{2}} \sum_{\mathrm{i}=1}^{\mathrm{N}} \sum_{\mathrm{j}=1}^{\mathrm{N}}\left(\mathrm{Z}_{\mathrm{ij}}-\overline{\mathrm{Z}}_{\mathrm{j} j}\right)^{2}$

where $Z_{i j}$ are the $Z$-coordinates of the analytical description of a sphere and a cone, $\mathrm{Z}_{\mathrm{ij}}$ are the $\mathrm{Z}$-coordinates obtained by the recovering of the images.

Table 2 presents the error in the calculating of the Mean $(\mathbf{H})$ and Gaussian $(\mathbf{K})$ curvature

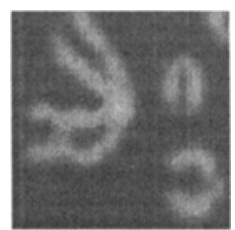

Fig.4a.

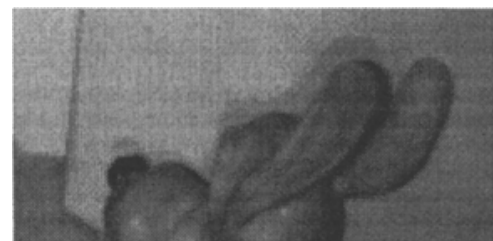

Fig.4b.

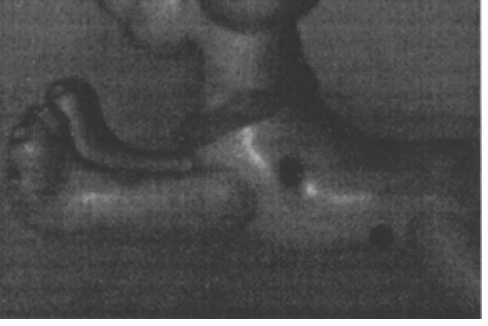

Input images

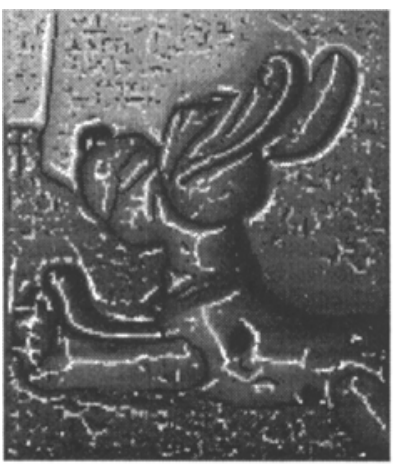

a

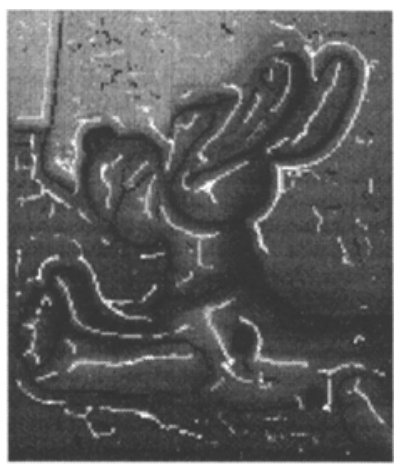

b

Fig.5. The ridges (white points) and the valley (black points). 
Table 1: The error in the recovering of the $\mathrm{Z}$ coordinate

\begin{tabular}{|l|c|c|}
\hline Method & $\begin{array}{c}\text { Sphere } \\
\bar{\varepsilon}_{\mathrm{z}}{ }^{2} \%\end{array}$ & $\begin{array}{c}\text { Cone } \\
\bar{\varepsilon}_{\mathrm{z}}{ }^{2} \%\end{array}$ \\
\hline As 2.1 & 0.68 & 0.57 \\
\hline As 2.2 & 0.56 & 0.45 \\
\hline
\end{tabular}

Table 2: The error in the calculating of the Mean (H) and Gaussian (K) curvature

\begin{tabular}{|l|r|r|r|r|}
\hline \multirow{2}{*}{ Object } & \multicolumn{2}{|c|}{ Sphere } & \multicolumn{2}{c|}{ Cone } \\
\cline { 2 - 5 } Method & $\bar{\varepsilon}_{\mathrm{K}}^{2} \%$ & $\bar{\varepsilon}^{2}{ }_{\mathrm{H}} \%$ & $\bar{\varepsilon}_{\mathrm{K}}^{2} \%$ & $\bar{\varepsilon}_{\mathrm{H}}^{2} \%$ \\
\hline As 2.1 & 2.45 & 1.22 & 2.10 & 1.01 \\
\hline As 2.2 & 1.18 & 0.5 & 0.75 & 0.32 \\
\hline
\end{tabular}
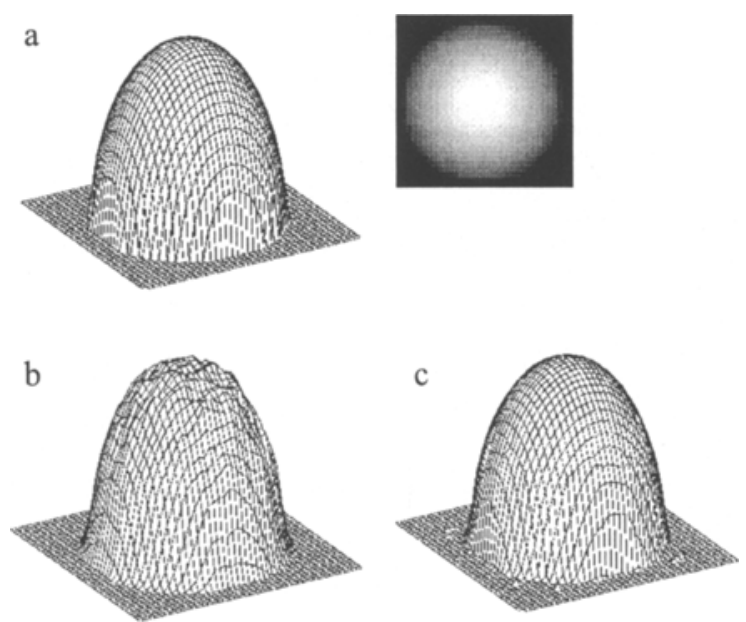

Fig.6. Comparison between the results obtained using the 2 methods 

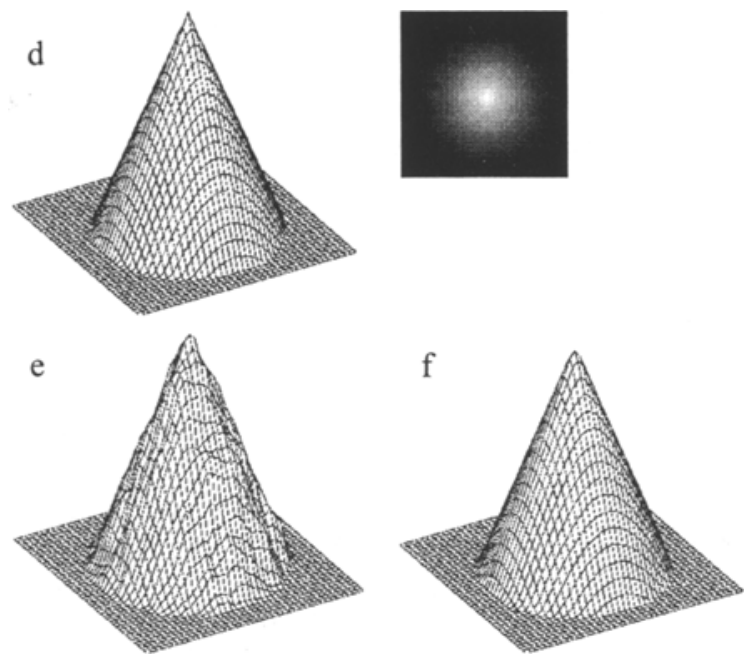

Fig.6. Comparison between the results obtained using the 2 methods

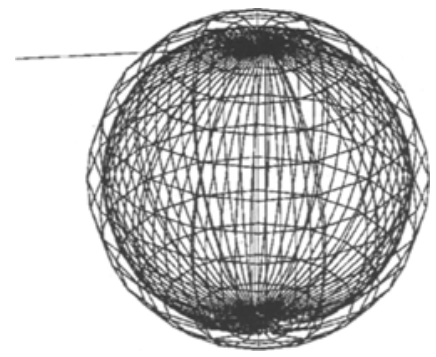

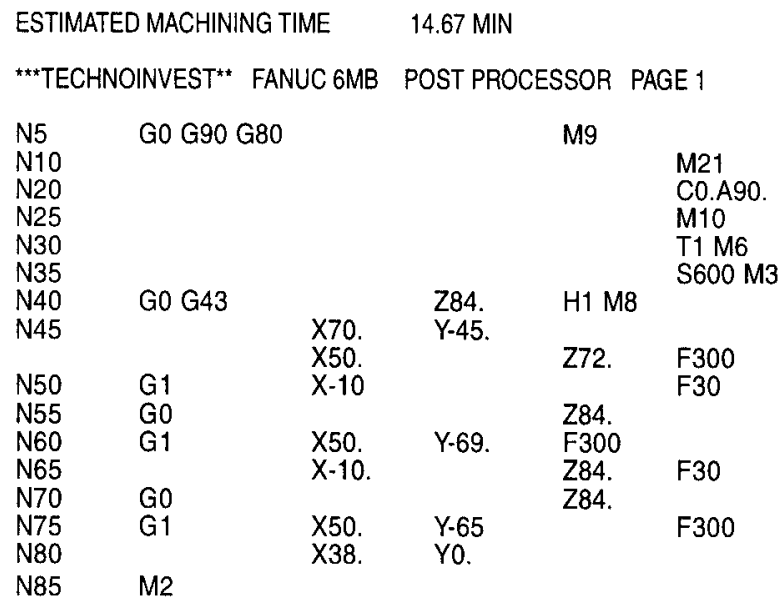

Fig.7. The tool path and part of the CNC program for the manufacturing of a sphere 
Figure 6 a,d show the objects (a sphere and a cone) described analytically.

Figure 6 b,e show the objects (a sphere and a cone) recovered using the method described in 2.1

Figure $6 \mathrm{c}, \mathrm{f}$ show the objects (a sphere and a cone) recovered using the method described in 2.2

Figure 7 shows the tool path and part of the CNC program for the manufacturing of a sphere.

Our work is realized using by a program on the language of $C$. Parts of it are adopted in Bulgarian manufactures.

\section{Conclusions}

1. The present paper is a theoretical and program-like research of two separate subsystems as a superstructure of the existing systems for designing components in the manufacturing (CAD/CAM systems):

1. A subsystem for automatic input of the geometrical description of visible surfaces from grey images in the CAD system has been worked out.

2. A new CAM subsystem for generating of CNC programs for metal cutting machines for processing of complex components has been worked out. The input of the system is the information aboul 3-dimensional scenes, received from a TV camera and as an output it produces a CNC program.

2. Method for the approximaling of a net of points with a rectangular net of curves have been achieved. The originally new items in the so presented method are the following:

- the choice of a net of curves for presenting the surface.

- the choice of the cubic interpolation splines as approximating curves for the surface.

- the approximation of every following point with a point from the cubic interpolation splines.

\section{References}

1. BesI P. and R. Jain. (1985) Three-Dimensional Object Recognition. Computing Surveys, Vol.17, Nil, pp.75-140.

2. Shirai Y., (1988) Three Dimensional Computer Vision.

3. Berthold K., P. Horn. (1993) Impossible Shaded Images. IEEE Trans. Patl. Anal. Machine Intell., vol. 15, №2.

4. Bhandarkar S.M. and Andreas Siebert. (1992) Integrating edge surface information for range image segmentation. Pattern Recognition, Vol.25, № 9, pp. 947-962.

5. Haralick R. (1983) Ridge and Valleys on Digital Images. Computer Vision, Graphics and Image Processing, 22 pp.28-38.

6. Kim and Biegel. (1988) A path generation method for sculplured surface manufacture. Computer ind. Engng., 14, pp. 95-101.

7. Milanova M., Alexandrov I., (1995)Recovering and RepresentationThree Dimensional Objects for Computer Vision or Computer Graphic Applications. Proc. of the International Conference on Digital Signal Processing, Cyprus. 